\title{
Ultracompact high-efficiency polarizing beam splitter with a hybrid photonic crystal and conventional waveguide structure
}

\author{
J. Cai \\ S. Kim \\ Gregory P. Nordin \\ nordin@byu.edu \\ J. Jiang
}

Follow this and additional works at: https://scholarsarchive.byu.edu/facpub

Part of the Electrical and Computer Engineering Commons

\section{Original Publication Citation}

S. Kim, G. P. Nordin, J. Cai, and J. Jiang, "Ultracompact high-efficiency polarizing beam splitter with a hybrid photonic crystal and conventional waveguide structure," Opt. Lett. 28(23), pp.

2384-2386 (23)

\section{BYU ScholarsArchive Citation}

Cai, J.; Kim, S.; Nordin, Gregory P.; and Jiang, J., "Ultracompact high-efficiency polarizing beam splitter with a hybrid photonic crystal and conventional waveguide structure" (2003). Faculty Publications. 1047. https://scholarsarchive.byu.edu/facpub/1047 accepted for inclusion in Faculty Publications by an authorized administrator of BYU ScholarsArchive. For more information, please contact ellen_amatangelo@byu.edu. 


\title{
Ultracompact high-efficiency polarizing beam splitter with a hybrid photonic crystal and conventional waveguide structure
}

\author{
Seunghyun Kim, Gregory P. Nordin, Jingbo Cai, and Jianhua Jiang \\ Laboratory for Integrated Computing and Optoelectronic Systems, University of Alabama in Huntsville, Huntsville, Alabama 35899
}

Received June 24, 2003

\begin{abstract}
We propose an ultracompact high-efficiency polarizing beam splitter that operates over a wide wavelength range and is based on a hybrid photonic crystal and a conventional waveguide structure. Within a small area $(15 \mu \mathrm{m} \times 10 \mu \mathrm{m})$, this polarizing beam splitter separates TM- and TE-polarized modes into orthogonal output waveguides. Results of simulations with the two-dimensional finite-difference time-domain method show that $99.3 \%$ of TM-polarized light is deflected by the photonic crystal structure (with a $28.0-\mathrm{dB}$ extinction ratio), whereas $99.0 \%$ of TE-polarized light propagates through the structure (with a 32.2-dB extinction ratio). Wave vector diagrams are employed to explain the operation of a polarizing beam splitter. Tolerance analysis reveals a large tolerance to fabrication errors. (c) 2003 Optical Society of America

OCIS codes: $230.5440,230.3120,230.7370,230.3990,250.5300$.
\end{abstract}

The use of hybrid photonic crystal (PhC) and conventional waveguide (CWG) structures was recently proposed as a way to achieve ultracompact planar lightwave circuits. ${ }^{1}$ Hybrid structures are formed by integration of a two-dimensional $\mathrm{PhC}$ region with CWGs to manipulate the direction of light propagation in a very small area (lateral dimension of the order of the waveguide mode size). In this Letter we present the numerical design and analysis of an ultracompact high-efficiency polarizing beam splitter with a high extinction ratio. Numerous approaches to achieving waveguide polarizing beam splitters have been reported in the literature ${ }^{2-6}$; they typically require relatively long waveguide structures (length of the order of millimeters). The approach presented in this Letter occupies an area of only $15 \mu \mathrm{m} \times 10 \mu \mathrm{m}$. Moreover, we show that its performance accommodates reasonable fabrication tolerances.

The geometry of a high-efficiency $\mathrm{PhC}-\mathrm{CWG}$ polarizing beam splitter is shown in Fig. 1(a). Light incident from the left is split into vertical and horizontal output waveguides according to its polarization (vertical, TM; horizontal, TE; TM and TE are the electric fields out of plane and in plane, respectively). The waveguides are designed to be single mode for wavelengths near $1.55 \mu \mathrm{m}\left(2-\mu \mathrm{m}\right.$ width, $n_{\text {core }}=1.500$, $\left.n_{\text {clad }}=1.465\right)$. The $\mathrm{PhC}$ is composed of a square array of silicon posts $(n=3.481)$. The boundaries between the $\mathrm{PhC}$ region and the CWGs are created by cuts in the $\Gamma-\mathrm{M}$ direction of the $\mathrm{PhC}$, which form an angle of $45^{\circ}$ with the input waveguide. The lattice constant and the radius of the $\mathrm{Si}$ posts of the $\mathrm{PhC}$ are 380 and $86.8 \mathrm{~nm}$, respectively.

The spectral response of this structure for both TMand TE-polarized incident light is shown in Fig. 1(b) as calculated by the finite-difference time-domain (FDTD) method ${ }^{7}$ with Berenger's perfectly matched layer boundary conditions. ${ }^{8}$ In the simulations a single-mode source is used to launch TM or TE light into the input waveguide and a Poynting vector calculation is used to monitor the optical power in the output waveguides, which is divided by the incident power to yield the efficiency with which light is directed into the output waveguides. From Fig. 1(b) it is clear that this hybrid structure splits TM and TE polarization to the output waveguides effectively for a wide wavelength range. The waveguide inside the $\mathrm{PhC}$ region is designed to follow the direction of propagation of the TE-polarized light, which leads to a $0.75-\mu \mathrm{m}$ shift of the horizontal output waveguide in the $-y$ direction from the input waveguide, as shown in Fig. 1(a).

The magnitude squared of the time-averaged electric field (TM) and magnetic field (TE) at $\lambda=1.55 \mu \mathrm{m}$ are shown in Figs. 2(a) and 2(b), respectively. TMpolarized light is reflected with $99.3 \%$ efficiency at the $\mathrm{PhC}$ surface and guided along the vertical output waveguide; $0.06 \%$ of the incident light passes through the $\mathrm{PhC}$ region. In TE-polarized light, $99.0 \%$ passes through the $\mathrm{PhC}$ region into the horizontal waveguide and $0.16 \%$ is reflected into the vertical output waveguide. The TM and TE output extinction ratios are 28.0 and $32.2 \mathrm{~dB}$, respectively.

Wave-vector (i.e., equifrequency) diagrams are useful as an aid in understanding how the hybrid structure operates. In Fig. 3, wave-vector diagrams for TM and TE polarization are shown for $\lambda=1.55 \mu \mathrm{m}$. The horizontal axes of the wave-vector diagrams correspond to the $\Gamma-\mathrm{M}$ direction of the $\mathrm{PhC}$, which forms the incident interface of the $\mathrm{PhC}$. The square and diamond insets in Figs. 1 and 3, which indicate the first Brillouin zone of the $\mathrm{PhC}$, show how the physical geometry is related to the wave vector diagram. In Fig. 3, the solid semicircles in the upper half-space are allowed wave vectors in the non- $\mathrm{PhC}$ region (assuming that this region is quasi-homogeneous with a refractive index of 1.485) and the dotted curves are the wave vectors of allowed propagation modes in the $\mathrm{PhC}$. The solid arrows denote propagation directions of the incident, reflected, and refracted light. The propagation direction of the refracted light in the $\mathrm{PhC}$ is determined by the group-velocity direction, which is calculated by the gradient of the dispersion 


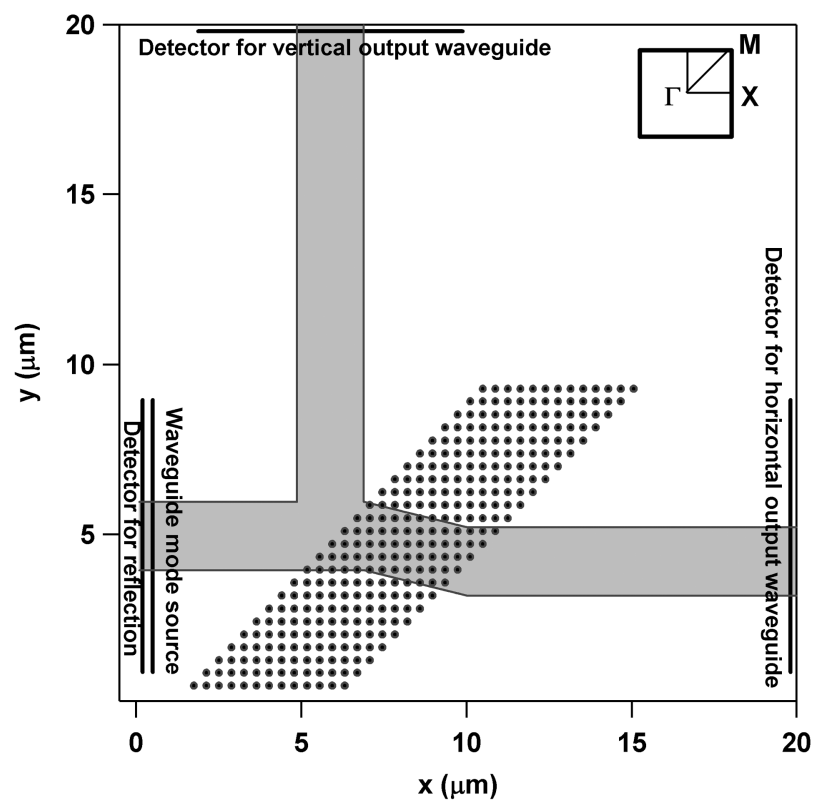

(a)

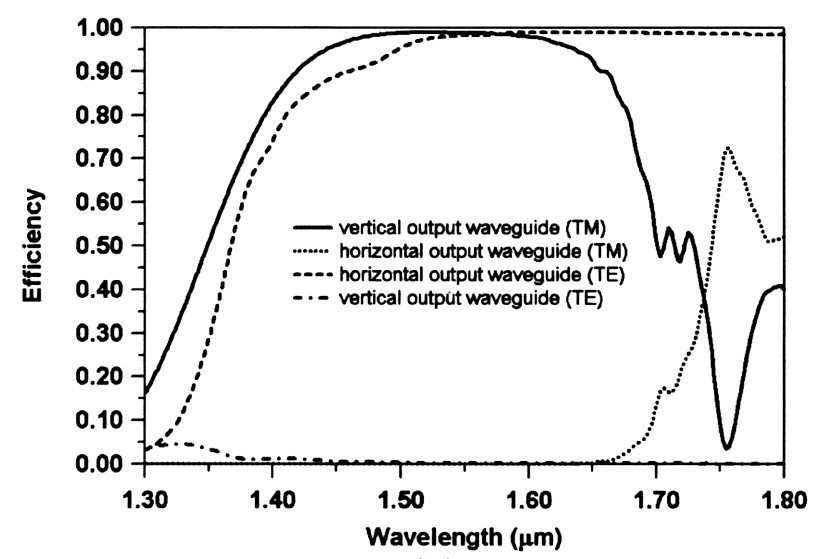

(b)

Fig. 1. (a) Geometry of a polarizing beam splitter composed of a square array of $\mathrm{Si}$ posts embedded in waveguides. The waveguide mode source line and detector lines for efficiency calculations are indicated. Square inset, first Brillouin zone of the PhC. (b) Efficiencies as a function of wavelength for TE- and TM-polarized incident light calculated at the detectors on the vertical and horizontal output waveguides as indicated in (a).

surface. $^{9,10}$ The grating vector associated with the periodicity at the boundary of the $\mathrm{PhC}$ is represented by the dashed arrows in Fig. 3.

From Fig. 3(a) we can easily see that there is no possible mode in the $\mathrm{PhC}$ region for TM-polarized light incident along the input waveguide at a $45^{\circ}$ angle to the $\mathrm{PhC}$ surface. Moreover, there are no allowed diffraction orders from diffraction by the periodic $\mathrm{PhC}$ boundary. Therefore, only zero-order reflection is allowed for light incident upon the $\mathrm{PhC}$ boundary, and this reflected light couples into the vertical output waveguide. TE-polarized light at $45^{\circ}$ incidence to the $\mathrm{PhC}$ boundary, however, has a possible propagation mode in the PhC region, although there are still no allowed diffraction orders, as shown in Fig. 3(b). Because the allowed wave vectors in the $\mathrm{PhC}$ for $\mathrm{TE}$ polarization form a semicircle, the $\mathrm{PhC}$ region can be considered an effective homogeneous material. The effective index calculated from the wave-vector diagram in Fig. 3(b) is 1.682. This value is smaller than the effective index calculated from the area-weighted average index of the $\mathrm{PhC}$ because the field propagating through the $\mathrm{PhC}$ region is localized primarily in the low-index material.

Using the effective index and considering the PhC region as an isotropic material, we can explain why 99.0\% of TE-polarized light can be coupled into the horizontal output waveguide. The Brewster angle calculated for the PhC's effective index is $48.56^{\circ}$, which is close to the incident angle $\left(45^{\circ}\right)$. The reflections at both interfaces are therefore very small $[0.015 \%$ for Fresnel reflection calculated with effective indices of the non- $\mathrm{PhC}$ region (1.485) and the $\mathrm{PhC}$ region (1.682)]. Because the reflections at both interfaces are so small, high coupling efficiency into the horizontal output waveguide for TE-polarized light is obtained.

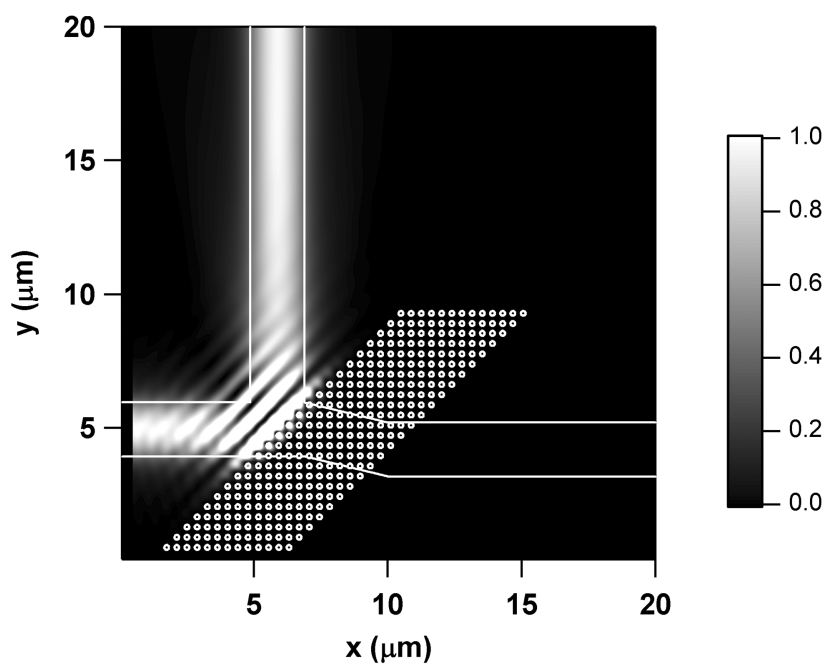

(a)

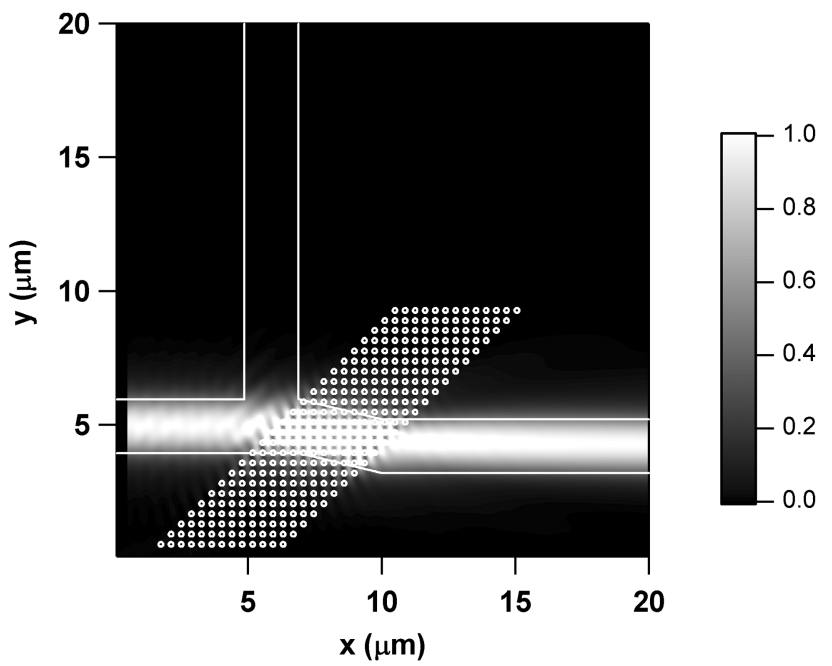

(b)

Fig. 2. Magnitudes squared of time-averaged (a) electric field (TM) and (b) magnetic field (TE) at $\lambda=1.55 \mu \mathrm{m}$ calculated by the two-dimensional FDTD method. The Yee cell size in the FDTD simulation is $10 \mathrm{~nm}$. 


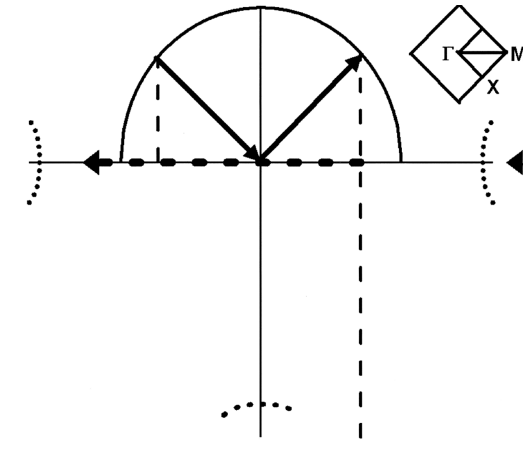

(a)

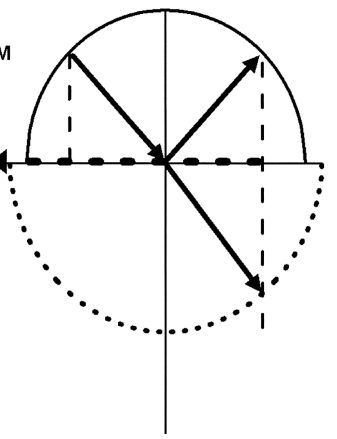

(b)
Fig. 3. Wave-vector diagrams for a polarizing beam splitter at $\lambda=1.55 \mu \mathrm{m}$. Diamond insets, first Brillouin zone with respect to the wave-vector diagram: (a) TM (after Ref. 1), (b) TE.

In addition, we examined the performance of the polarizing beam splitter as a function of potential fabrication errors that affect Si-post radii and periods and positional and angular misalignment of the $\mathrm{PhC}$ region with respect to the waveguides over the wavelength range 1.53-1.62 $\mu \mathrm{m}(C$ and $L$ bands for optical communication). Results for a variation in Si-post radii from 80.8 to $90.8 \mathrm{~nm}$ (20-nm range for the diameter) show that the TM efficiency varies from $93.0 \%$ to $99.3 \%$, whereas the corresponding range for the $\mathrm{TE}$ efficiency is 94.5-99.0\%. At a wavelength of $1.55 \mu \mathrm{m}$ the efficiency for the TMpolarized light at the vertical output waveguide is in the range 98.7-99.3\%, whereas more than $97.2 \%$ of TE-polarized light is coupled into the horizontal output waveguide. The efficiencies of TM- and TE-polarized modes are greater than $94 \%$ for changes of the $\mathrm{PhC}$ period from 370 to $400 \mathrm{~nm}$ over the $C$ and $L$ bands. Also, the TM and TE efficiencies are greater than 95\% for $\pm 0.5-\mu \mathrm{m}$ misalignment of the $\mathrm{PhC}$ region in both the $x$ and the $y$ directions. A PhC angular misalignment greater than $\pm 1^{\circ}$ is required before the TM efficiency decreases $95 \%$ over the wavelength range. Actual fabrication angular misalignments are of course much smaller than a degree. Our tolerance simulations show that the proposed polarizing beam splitter design has reasonable fabrication tolerances for operation over the $C$ and $L$ bands.

In conclusion, we have proposed an ultracompact (15 $\mu \mathrm{m} \times 10 \mu \mathrm{m}$ area) polarizing beam splitter design with high efficiency (99.3\% for TM-polarized light and 99.0\% for TE-polarized light) and a high extinction ratio (28.0 dB for TM polarization and $32.2 \mathrm{~dB}$ for TE polarization) composed of a PhC embedded in low-index contrast CWGs. The proposed polarizing beam splitter has reasonable tolerances to fabrication errors.

This research was supported in part by Defense Advanced Research Projects Agency grant N66001-01-1-8938 and National Science Foundation grant EPS-0091853. G. P. Nordin's e-mail address is nordin@ece.uah.edu.

\section{References}

1. G. P. Nordin, S. Kim, J. Cai, and J. Jiang, Opt. Express 10, 1334 (2002), http://www.opticsexpress.org.

2. R. M. de Ridder, A. F. M. Sander, A. Driessen, and J. H. J. Fluitman, J. Lightwave Technol. 11, 1806 (1993).

3. P. Wei and W. Wang, IEEE Photon. Technol. Lett. 6, 245 (1994).

4. L. B. Soldano, A. H. de Vreede, M. K. Smit, B. H. Verbeek, E. G. Metaal, and F. H. Groen, IEEE Photon. Technol. Lett. 6, 402 (1994).

5. H. Maruyama, M. Haruna, and H. Nishihara, J. Lightwave Technol. 13, 1550 (1995).

6. S. M. Garner, V. Chuyanov, S. Lee, A. Chen, W. H. Steier, and L. R. Dalton, IEEE Photon. Technol. Lett. 11, 842 (1999).

7. A. Taflove, Computational Electrodynamics: The Finite-Difference Time-Domain Method (Artech House, Boston, Mass., 1995).

8. J. P. Berenger, J. Comput. Phys. 114, 185 (1994).

9. M. Notomi, Phys. Rev. B 62, 10,696 (2000).

10. T. Baba and M. Nakamura, IEEE J. Quantum Electron. 38, 909 (2002). 